\begin{tabular}{|c|c|}
\hline Citation/Reference & $\begin{array}{l}\text { de la Hucha Arce F., Patrinos P., Verhelst M., Bertrand A., } \\
\text { On the convexity of bit depth allocation for linear } \\
\text { MMSE estimation in wireless sensor networks, in } \\
\text { IEEE Signal Processing Letters, vol. 27, pp. 291-295, } 2020 .\end{array}$ \\
\hline Archived version & Author's version after peer review \\
\hline Published version & $\begin{array}{l}\underline{\text { http://ieeexplore.ieee.org/stamp/stamp.jsp? }} \\
\underline{\text { tp }=\& a r n u m b e r=8962239 \& i s n u m b e r=8966529} \\
\text { DOI: } \underline{\text { https://doi.org/10.1109/LSP.2020.2967592 }}\end{array}$ \\
\hline Journal homepage & $\begin{array}{l}\text { https://signalprocessingsociety.org/publications- } \\
\text { resources/ieee-signal-processing-letters }\end{array}$ \\
\hline Author contact & $\begin{array}{l}\text { Email: fernando.delahuchaarce@esat.kuleuven.be } \\
\text { Phone number: + } 32(0) 16324567\end{array}$ \\
\hline Abstract & $\begin{array}{l}\text { Energy efficiency is crucial for a wireless sensor network (WSN) since its } \\
\text { nodes are generally powered by energy sources of limited capacity, such } \\
\text { as batteries. The bit depth used to quantize the sensor signal samples } \\
\text { heavily influences energy consumption, as it strongly impacts the amount } \\
\text { of information to be transmitted between the sensor nodes. Bit depth } \\
\text { allocation problems seek to assign a certain bit depth to each sensor } \\
\text { signal such that energy consumption is minimized while respecting a } \\
\text { performance constraint. For multi-channel signal estimation tasks these } \\
\text { problems are generally non-convex, and they are often solved through } \\
\text { simplifying assumptions or through convex relaxation. However, for linear } \\
\text { minimum mean squared error (MMSE) estimation, we show how the } \\
\text { matrix inversion lemma allows to transform the MMSE constraint into a } \\
\text { convex constraint, which can then be interpreted as a constraint on the } \\
\text { excess MMSE due to quantization. As a result, as long as the cost } \\
\text { function representing energy consumption is convex, this class of bit depth } \\
\text { allocation problems is convex, i.e., if the bit depth variable is relaxed to a } \\
\text { real-valued variable. This guarantees global optimality up to discretization } \\
\text { of the obtained solution. }\end{array}$ \\
\hline IR & $\mathrm{N} / \mathrm{a}$ \\
\hline
\end{tabular}




\title{
On the convexity of bit depth allocation for linear MMSE estimation in wireless sensor networks
}

\author{
Fernando de la Hucha Arce, Panagiotis Patrinos, Member, IEEE, Marian Verhelst, Senior Member, IEEE, and \\ Alexander Bertrand, Senior Member, IEEE
}

\begin{abstract}
Energy efficiency is crucial for a wireless sensor network (WSN) since its nodes are generally powered by energy sources of limited capacity, such as batteries. The bit depth used to quantize the sensor signal samples heavily influences energy consumption, as it strongly impacts the amount of information to be transmitted between the sensor nodes. Bit depth allocation problems seek to assign a certain bit depth to each sensor signal such that energy consumption is minimized while respecting a performance constraint. For multi-channel signal estimation tasks these problems are generally non-convex, and they are often solved through simplifying assumptions or through convex relaxation. However, for linear minimum mean squared error (MMSE) estimation, we show how the matrix inversion lemma allows to transform the MMSE constraint into a convex constraint, which can then be interpreted as a constraint on the excess MMSE due to quantization. As a result, as long as the cost function representing energy consumption is convex, this class of bit depth allocation problems is convex, i.e., if the bit depth variable is relaxed to a real-valued variable. This guarantees global optimality up to discretization of the obtained solution.
\end{abstract}

Index Terms-Bit depth allocation, energy efficiency, signal estimation, wireless sensor networks

\section{INTRODUCTION}

A wireless sensor network (WSN) is a collection of sensor nodes which exchange their observations of a physical phenomenon using wireless communications. The energy budget of the sensor nodes is usually limited since they are typically powered by batteries, which may be difficult to replace or recharge. As a consequence, energy efficiency is crucial in the design of algorithms for WSNs. In particular, data exchange through wireless communications is generally more expensive than data processing at the sensor nodes [1]. One parameter that heavily affects the amount of data to be exchanged between the sensor nodes is the bit depth used to quantize the sensor signal samples. The goal of a bit depth allocation problem is to optimize the bit depth of each sensor signal such that the total communication energy consumption is minimized while respecting a performance constraint depending on the task assigned to the WSN. We focus on multi-channel signal estimation, which has applications in, e.g., speech enhance-

This research work was carried out at the ESAT Laboratory of KU Leuven in the frame of Research Fund KU Leuven C14/16/057, FWO nr. G.0931.14, the European Research Council (ERC) under the European Union's Horizon 2020 research and innovation programme (grant agreements No. 802895 and No. 715037). The scientific responsibility is assumed by the authors.

The authors are with the Dept. of Electrical Engineering (ESAT), Kasteelpark Arenberg 10, 3001 Leuven, Belgium, email: \{fernando.delahuchaarce, panos.patrinos, marian.verhelst, alexander.bertrand\}@esat.kuleuven.be.

Copyright (c) 2020 IEEE. Personal use is permitted, but republication/redistribution requires IEEE permission. ment for wireless acoustic sensor networks [2]-[5] and artifact removal in electroencephalography (EEG) networks [6].

Previous work in bit depth allocation problems for WSNs has been conducted for a variety of estimation algorithms. The works presented in [7], [8] propose several solutions to this class of problems in a WSN whose task is linear MMSE estimation. However, the authors assume that the noise sensor signal components are spatially uncorrelated, which precludes its use in many practical applications where noise is spatially correlated, e.g., when localized noise sources are present. The effect of noise correlation is studied in [9], although for a suboptimal estimator. Spatial correlation of the desired sensor signal components following a specific mathematical model is considered in [10], where the noise sensor signal components are again assumed uncorrelated. In [11], [12] several bit depth allocations problems are solved for multihop WSNs where each node averages the estimators from its neighbours instead of computing the true linear MMSE estimator. In [13], [14] a greedy bit depth allocation algorithm is proposed, but its convergence speed is low when steps of one bit must be used. In [15], a bit depth allocation problem based on a linearly constrained minimum variance (LCMV) estimator is solved through convex relaxation of the minimum variance constraint, thereby loosing guarantees on the optimality of the solution.

In this paper we focus on bit depth allocation problems based on a linear MMSE signal estimation task for a WSN, with a general signal model that considers correlated noise components across the different sensor nodes. We assume a centralized architecture where all sensor signals are transmitted to a fusion centre (FC). We show that, while a constraint on MMSE is non-convex in general, it can be transformed into a convex constraint using a similar strategy as in [15], which can then be interpreted as a constraint on the excess MMSE due to quantization. Therefore, as long as the cost function representing energy consumption is convex, this class of bit depth allocation problems is convex. This guarantees global optimality of the solution up to discretization.

The remaining of the paper is organized as follows. In Section II we formulate the bit depth allocation problem. In Section III we transform the MMSE constraint into a convex constraint. In Section IV we provide numerical simulations. Finally, we present our conclusions in Section V.

\section{Bit DEPTH ALLOCATION BASED ON MMSE}

\section{A. Signal model}

We consider a WSN composed of $K$ sensors (channels), where the set of sensors is denoted by $\mathcal{K}=\{1, \ldots, K\}$. 
The signal samples collected by the $k$-th sensor are denoted by $y_{k}[t]$, where $t \in \mathbb{N}$ denotes the discrete time index. For conciseness, we will omit the time index in the subsequent sections unless referring to a specific signal sample. We assume that the signals $y_{k}$ are complex-valued in order to allow frequency domain descriptions such as, e.g., the short-time Fourier transform (STFT). Furthermore, all sensor signals are assumed to be realizations of short-term wide-sense stationary and short-term ergodic stochastic processes.

The sensor signal $y_{k}$ is modelled as the combination of a desired signal component $x_{k}$ and an undesired zero-mean noise component $v_{k}$, which can be expressed as

$$
y_{k}=x_{k}+v_{k}, \quad k \in\{1, \ldots, K\},
$$

where we assume that $E\left\{x_{k} v_{m}^{*}\right\}=0 \forall k, m \in \mathcal{K}$, where $E\{\cdot\}$ is the expectation operator. The sensor signals can be stacked in the $K \times 1$ vector $\mathbf{y}=\left[y_{1}, \ldots, y_{K}\right]^{T}$. The $K \times 1$ vectors $\mathbf{X}$ and $\mathbf{v}$ are defined in a similar way. We remark that no statistical distribution, Gaussian or otherwise, is assumed on the sensor signals $y_{k}$ or their components $x_{k}, v_{k}$.

\section{B. Linear MMSE estimation}

For the sake of simplicity, we assume a centralized architecture for the WSN where all sensors send their signal samples to a fusion centre (FC), which then has access to the complete set of sensor signals $\mathbf{y}$.

The goal of the WSN is to estimate a desired signal $d$, which corresponds to any one of the unobservable desired signal components, i.e., $d=x_{k}$ for some $k$, by applying the linear MMSE estimator to the sensor signals $y$. The linear MMSE estimator $\hat{\mathbf{w}}$ is found by minimizing the mean squared error cost function, i.e.,

$$
\hat{\mathbf{w}}=\underset{\mathbf{w}}{\arg \min } E\left\{\left|d-\mathbf{w}^{H} \mathbf{y}\right|^{2}\right\},
$$

where $(\cdot)^{H}$ denotes the conjugate transpose. Assuming that the sensor signal correlation matrix $\mathbf{R}_{y y}=E\left\{\mathbf{y} \mathbf{y}^{H}\right\}$ has full $\operatorname{rank}^{1}$, the unique solution to (2) is given by

$$
\hat{\mathbf{w}}=\mathbf{R}_{y y}^{-1} \mathbf{r}_{y d},
$$

where $\mathbf{r}_{y d}=E\left\{\mathbf{y} d^{*}\right\}$. The estimate of the desired signal $d$ is then given by

$$
\hat{d}=\hat{\mathbf{w}}^{H} \mathbf{y} .
$$

The linear minimum mean squared error is given by

$$
E\left\{\left|d-\hat{\mathbf{w}}^{H} \mathbf{y}\right|^{2}\right\}=P_{d}-\mathbf{r}_{y d}^{H} \mathbf{R}_{y y}^{-1} \mathbf{r}_{y d} .
$$

where $P_{d}=E\left\{|d|^{2}\right\}$ is the desired signal power.

Using the assumption of short-term ergodicity, the matrix $\mathbf{R}_{y y}$ can be estimated through sample averaging, e.g., using a sliding window or a forgetting factor. As the desired signal $d$ is not observable, sample averaging is impossible for $\mathbf{r}_{y d}$, and hence its estimation has to be done indirectly through strategies specific to each application. In cases where the desired source signal has an 'on-off' behaviour, as in speech

\footnotetext{
${ }^{1}$ This assumption is typically satisfied in practice due to the presence of a noise component in each sensor that is independent of other sensor signals.
}

[3]-[5] or EEG signal enhancement [6], the noise correlation matrix $\mathbf{R}_{v v}=E\left\{\mathbf{v} \mathbf{v}^{H}\right\}$ can be estimated during periods when the desired source is inactive, which allows to obtain $\mathbf{r}_{y d}$ from the estimates of $\mathbf{R}_{v v}$ and $\mathbf{R}_{y y}$ [3]. More refined data-driven techniques to estimate $\mathbf{r}_{y d}$ have been studied in [16], [17].

\section{Uniform quantization}

In practical applications of WSNs, the samples of each sensor signal $y_{k}$ are quantized prior to their transmission using a certain bit depth $b_{k}$, which introduces a quantization error $e_{k}{ }^{2}$. The uniform quantization of $y_{k}$ with $b_{k}$ bits can be expressed as

$$
Q\left(y_{k}\right)=\Delta_{b_{k}}\left(\left\lfloor\frac{y_{k}}{\Delta_{b_{k}}}\right\rfloor+\frac{1}{2}\right),
$$

where $\lfloor\cdot\rfloor$ denotes the floor function and $\Delta_{b_{k}}=D_{k} / 2^{b_{k}}$. The parameter $D_{k}$ is the dynamic range of the sensor signal $y_{k}$, which is typically selected to be a few standard deviations around its mean or $2 \max \left(\left|y_{k}\right|\right)$. Since the dynamic range is specific to each sensor signal, it needs to be communicated to the FC. The quantization error is then defined as

$$
e_{k}=Q\left(y_{k}\right)-y_{k} \text {. }
$$

The mathematical properties of the quantization error $e_{k}$ have been thoroughly studied [18]-[20]. It has been shown that the quantization error is zero mean, and that $y_{k}$ and $e_{k}$ are uncorrelated under certain technical conditions on the characteristic function of $y_{k}$ [18]. Under the same conditions, the power of the quantization error is given by

$$
p_{k}=E\left\{\left|e_{k}\right|^{2}\right\}=\frac{\Delta_{b_{k}}^{2}}{12}=\frac{D_{k}^{2}}{12} \frac{1}{2^{2 b_{k}}} .
$$

Following the uniform quantization model, we can express the quantized sensor signal samples of the $k$-th sensor as

$$
y_{k, e}=y_{k}+e_{k},
$$

which can then be stacked in the $K \times 1$ vector

$$
\mathbf{y}_{e}=\left[y_{1, e}, \ldots, y_{K, e}\right]^{T}=\mathbf{y}+\mathbf{e},
$$

where $\mathbf{e}=\left[e_{1}, \ldots, e_{K}\right]^{T}$. Using the statistical properties of $e_{k}$ we can find the second-order statistics of the quantized sensor signals. As $e_{k}$ is assumed to be uncorrelated with $d$, the cross-correlation remains unchanged, i.e.

$$
\mathbf{r}_{y_{e} d}=E\left\{(\mathbf{y}+\mathbf{e}) d^{*}\right\}=\mathbf{r}_{y d} \text {. }
$$

Similarly, the quantized sensor signal correlation matrix is given by

$$
\mathbf{R}_{y_{e} y_{e}}=E\left\{(\mathbf{y}+\mathbf{e})(\mathbf{y}+\mathbf{e})^{H}\right\}=\mathbf{R}_{y y}+\mathbf{R}_{e e},
$$

where $\mathbf{R}_{e e}$ is the error correlation matrix, given by

$$
\mathbf{R}_{e e}=E\left\{\mathbf{e e}^{H}\right\}=\operatorname{diag}(\mathbf{p}),
$$

where $\mathbf{p}=\left[p_{1}, \ldots, p_{K}\right]^{T}$ is the $K \times 1$ vector collecting the quantization error powers. Note that (12) and the diagonal structure of $\mathbf{R}_{e e}$ in (13) follow from the assumptions that the quantization errors $e_{k}$ are uncorrelated with every sensor signal $y_{m}$ and every other quantization error $e_{n}, \forall m, n \in \mathcal{K}, n \neq k$.

\footnotetext{
${ }^{2}$ Note that $y_{k}$ could be already quantized, e.g., when it is acquired by the analog-to-digital converter of the $k$-th sensor. In that situation, $e_{k}$ would represent the error from changing the bit resolution of $y_{k}$.
} 


\section{Bit depth allocation}

The bit depth used to quantize each signal $y_{k}$ affects the energy spent in communicating its samples to the FC, and comes with a performance penalty in terms of mean squared error (MSE). Using (3), (11) and (12) we can find the optimal linear MMSE estimator for the quantized sensor signals $\mathbf{y}_{e}$ as

$$
\hat{\mathbf{w}}_{e}=\underset{\mathbf{w}}{\arg \min } E\left\{\left|d-\mathbf{w}^{H} \mathbf{y}_{e}\right|^{2}\right\}=\mathbf{R}_{y_{e} y_{e}}^{-1} \mathbf{r}_{y d} .
$$

The MMSE corresponding to a specific set of bit depths $\mathbf{b}=$ $\left[b_{1}, \ldots, b_{K}\right]^{T}$, denoted by $J(\mathbf{b})$, can be found from (5) as

$$
J(\mathbf{b})=E\left\{\left|d-\hat{\mathbf{w}}_{e}^{H} \mathbf{y}_{e}\right|^{2}\right\}=P_{d}-\mathbf{r}_{y d}^{H} \mathbf{R}_{y_{e} y_{e}}^{-1} \mathbf{r}_{y d},
$$

where $\mathbf{R}_{y_{e} y_{e}}$ depends on $\mathbf{b}$ through (8), (12) and (13).

The bit depth allocation problem with an MMSE constraint can be then formulated as

$$
\begin{aligned}
\min _{\mathbf{b}} & \sum_{k=1}^{K}\left(E_{k}\left(b_{k}\right)\right)^{q} \\
\text { s.t. } & P_{d}-\mathbf{r}_{y d}^{H} \mathbf{R}_{y_{e} y_{e}}^{-1} \mathbf{r}_{y d} \leq \eta \\
& b_{k} \in \mathbb{Z}_{+}, \quad b_{k} \leq b_{\max }, \forall k,
\end{aligned}
$$

where $E_{k}\left(b_{k}\right)$ is a function modelling the energy spent in communicating the $k$-th sensor signal quantized using $b_{k}$ bits to the $\mathrm{FC}, b_{\max }$ is the maximum bit depth allowed, $\eta$ is a userdefined threshold on the allowed MSE, and $\mathbb{Z}_{+}$is the set of non-negative integers. Note that the cost function in (16) is the $q$-th power of the $L_{q}$-norm of the sensor energy consumption vector $\mathbf{E}=\left[E_{1}, \ldots, E_{K}\right]^{T}$, where $q \geq 1$ is an integer. The $L_{1}$-norm corresponds to the total energy consumption, while the $L_{\infty}$-norm corresponds to the maximum sensor energy consumption. Other values of $q$ correspond to tradeoffs between these two criteria [7], [11], [12], [15], [21]. As exhaustive integer search is impractical for all but the smallest of problems, we relax the bit depths $b_{k}$ to be non-negative real numbers, i.e., $b_{k} \in \mathbb{R}_{+}$. The solution then needs to be transformed back to $\mathbb{Z}_{+}$, e.g., through rounding as in [7], [11], [12], randomized rounding [15] or a greedy heuristic [10]. In Section III-B we provide details on suitable energy models.

\section{CONVEX FORMULATION OF THE PROBLEM}

The MMSE constraint in (16) can be written using (12) as

$$
P_{d}-\mathbf{r}_{y d}^{H}\left(\mathbf{R}_{y y}+\mathbf{R}_{e e}\right)^{-1} \mathbf{r}_{y d} \leq \eta .
$$

Unfortunately (17) is not convex but, as we show in the following, can be transformed into a convex constraint. To this end, we transform the constraint into a linear matrix inequality, based on a similar strategy as in [15] for LCMV estimation. However, [15] required an additional convex relaxation on the constraints to finally end up with a convex problem, which turns out to not be necessary for the MMSE framework.

\section{A. Transformation into a convex constraint}

We begin with applying a special case of the Woodbury identity [22], which states that

$$
(\mathbf{R}+\mathbf{D})^{-1}=\mathbf{R}^{-1}-\mathbf{R}^{-1}\left(\mathbf{R}^{-1}+\mathbf{D}^{-1}\right)^{-1} \mathbf{R}^{-1},
$$

where $\mathbf{R}$ and $\mathbf{D}$ are invertible matrices of the same dimensions. Applying (18) to (17) we obtain

$$
P_{d}-\mathbf{r}_{y d}^{H}\left(\mathbf{R}_{y y}^{-1}-\mathbf{R}_{y y}^{-1}\left(\mathbf{R}_{y y}^{-1}+\mathbf{R}_{e e}^{-1}\right)^{-1} \mathbf{R}_{y y}^{-1}\right) \mathbf{r}_{y d} \leq \eta,
$$

where we note that $\mathbf{R}_{e e}=\operatorname{diag}(\mathbf{p})$ following (13) and, being a diagonal square matrix with all non-zero entries, is invertible ${ }^{3}$. Expanding and rearranging (19) we find

$\mathbf{r}_{y d}^{H} \mathbf{R}_{y y}^{-1}\left(\mathbf{R}_{y y}^{-1}+\mathbf{R}_{e e}^{-1}\right)^{-1} \mathbf{R}_{y y}^{-1} \mathbf{r}_{y d} \leq \eta-\left(P_{d}-\mathbf{r}_{y d}^{H} \mathbf{R}_{y y}^{-1} \mathbf{r}_{y d}\right)$,

where the left-hand term represents the excess MMSE due to quantization, and the right-hand term represents the difference between the user-defined threshold $\eta$ and the MMSE obtained when all sensor signals $\mathbf{y}$ are quantized at maximum resolution ( $b_{\max }$ or higher), following (5). By using the Schur complement lemma [23] we can express (20) as

$$
\left[\begin{array}{cc}
\mathbf{R}_{y y}^{-1}+\mathbf{R}_{e e}^{-1} & \mathbf{R}_{y y}^{-1} \mathbf{r}_{y d} \\
\mathbf{r}_{y d}^{H} \mathbf{R}_{y y}^{-1} & \bar{\eta}
\end{array}\right] \succeq 0
$$

where $\bar{\eta}=\eta-\left(P_{d}-\mathbf{r}_{y d}^{H} \mathbf{R}_{y y}^{-1} \mathbf{r}_{y d}\right)$ is the threshold on the excess MMSE due to quantization. Following (8) and (13), the expression for $\mathbf{R}_{e e}^{-1}$ is

$\mathbf{R}_{e e}^{-1}=\operatorname{diag}\left(p_{1}^{-1}, \ldots, p_{K}^{-1}\right)=\operatorname{diag}\left(12 \frac{2^{2 b_{1}}}{D_{1}^{2}}, \ldots, 12 \frac{2^{2 b_{K}}}{D_{K}^{2}}\right)$.

As $\mathbf{R}_{e e}^{-1}$ is linear in $2^{2 b_{k}}$, (21) constitutes a linear matrix inequality (LMI) in the variable $u_{k}=2^{2 b_{k}}$, equivalent to

$$
\mathbf{R}_{0}+\sum_{k=1}^{K} u_{k} \frac{12}{D_{k}^{2}} \mathbf{E}_{k k} \succeq 0
$$

where $\mathbf{E}_{k k}$ is the $(K+1) \times(K+1)$ matrix with all zero elements except the $k$-th element of its diagonal, which is equal to one, and

$$
\mathbf{R}_{0}=\left[\begin{array}{cc}
\mathbf{R}_{y y}^{-1} & \mathbf{R}_{y y}^{-1} \mathbf{r}_{y d} \\
\mathbf{r}_{y d}^{H} \mathbf{R}_{y y}^{-1} & \bar{\eta}
\end{array}\right] .
$$

It is well known that LMIs define convex sets [23], thus showing that the MMSE constraint in (23) is convex after the relaxation $b_{k} \in \mathbb{R}_{+}$mentioned in Section II-D.

\section{B. Implications for bit depth allocation problems}

The bit depth allocation problem (16) can be expressed as

$$
\begin{aligned}
\min _{\mathbf{u}} & \sum_{k=1}^{K}\left(\tilde{E}_{k}\left(u_{k}\right)\right)^{q} \\
\text { s.t. } & \mathbf{R}_{0}+\sum_{k=1}^{K} u_{k} \frac{12}{D_{k}^{2}} \mathbf{E}_{k k} \succeq 0 \\
& \quad 1 \leq u_{k} \leq 2^{2 b_{\max }}, \forall k \in\{1, \ldots, K\},
\end{aligned}
$$

where $\tilde{E}_{k}\left(u_{k}\right)=E\left(2^{-1} \log _{2} u_{k}\right)$. Since $q \geq 1$, a sufficient condition for the convexity of the problem is that $\tilde{E}_{k}\left(u_{k}\right)$

\footnotetext{
${ }^{3}$ The case where one or more entries of $\mathbf{R}_{e e}$ are zero requires a version of the matrix inversion lemma (18) where $\mathbf{D}$ is not an invertible matrix.
} 


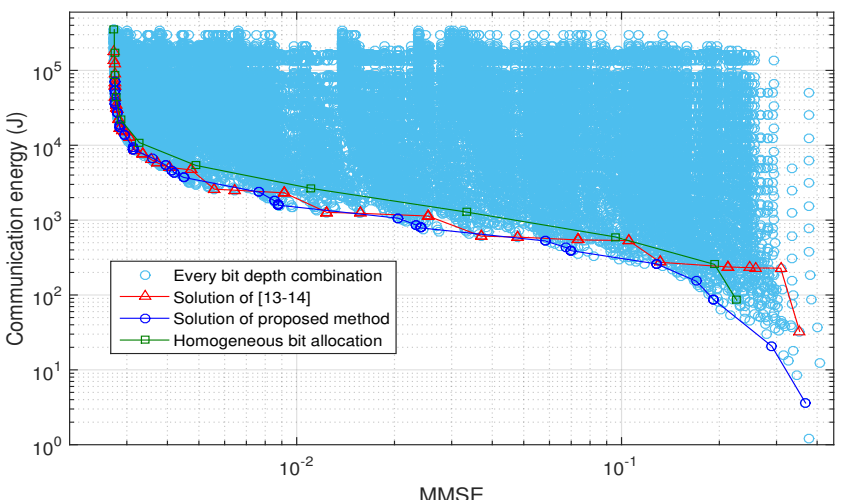

Figure 1. Energy and MMSE corresponding to every bit depth combination, the solution from the proposed algorithm, homogeneous allocation, and the greedy method of [13], [14] for $K=4$ sensors. The coordinates of the desired source are $[4.64,2.00]$ and of the noise sources $[1.89,3.94] ;[3.17,1.40]$

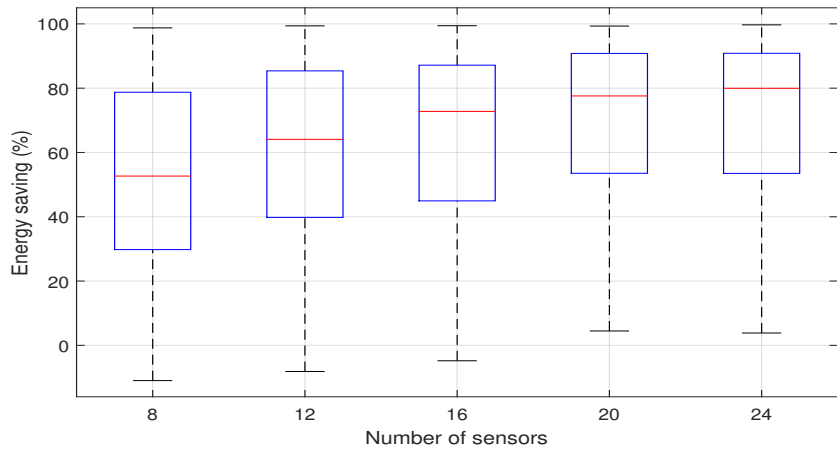

Figure 2. Energy savings of the proposed bit depth allocation method with respect to homogeneous allocation (same bit depth for every sensor).

are convex and non-negative $\forall k \in \mathcal{K}$. It can then be solved through efficient convex optimization methods such as the interior point method [23]. The $k$-th optimal bit depth $b_{k}^{*}$ can be recovered by $b_{k}^{*}=$ round $\left(2^{-1} \log _{2} u_{k}^{*}\right)$.

The function $E_{k}\left(b_{k}\right)=\rho_{k}\left(2^{\alpha b_{k}}-1\right), \alpha>0$, can be used as a generic model for additive white Gaussian noise (AWGN) communication channels between each sensor and the FC, which contains many energy models used in the literature as special cases. For example, in [11], [12], [21] the model is based on channel capacity leading to $\alpha \geq 1$, in [7] the assumption is made that the QAM constellation scales with the bit depth $b_{k}$ leading to $\alpha=1$, and in [15] it is again based on channel capacity with $\alpha=2$. The factors $\rho_{k} \geq 0$ are specific to the communication channel model of each of these works. Using this generic function for $E_{k}\left(b_{k}\right)$, the condition for the convexity of the cost function in (25) is

$$
q \geq 2 \alpha^{-1} \geq 1 \text {. }
$$

The derivation is provided in the Supplementary Material.

\section{Simulations}

We consider toy scenarios in a two dimensional $5 \times 5 \mathrm{~m}$ area where the positions of a single desired source, noise sources and sensors are randomly generated in 100 independent Monte Carlo runs. All source signals consist of $10^{5}$ samples of complex Gaussian white stochastic processes with zero mean and unit variance. We use a narrow-band propagation model where each source is observed at each sensor with a power attenuation factor inversely proportional to the distance between the corresponding source and the sensor, and a phase delay generated from a uniform distribution in $[-\pi, \pi]$. Besides, independent AWGN is also present in each sensor signal, with zero mean and a variance of $10^{-3}$ times the power of the corresponding sensor signal. The second-order statistics $\mathbf{R}_{y y}$ and $\mathbf{r}_{y d}$ are estimated by averaging over all the signal samples. As our interest lies in observing the effects of quantization, we compute $\mathbf{r}_{y d}$ assuming knowledge of the desired signal $d$ (the desired source as observed in the sensor with the highest SNR) to avoid estimation errors. The energy model is taken to be $E_{k}\left(b_{k}\right)=\rho_{k}\left(2^{b_{k}}-1\right)$, as described in (25), where we choose $\rho_{k}=r_{k}^{2}$, where $r_{k}$ is the distance between the $k$-th sensor and the FC, and $q=2$ for simplicity.

Figure 1 depicts, in a representative scenario with $K=4$ sensors and two noise sources, the resulting total energy and MMSE from the bit depth allocation (25) for different thresholds $\eta$, solved using the CVX package [24], [25]. Besides, it also depicts those magnitudes resulting from every bit depth combination from $b_{\max }=12$ to 0 bits, from homogeneous allocation, i.e., where every sensor uses the same bit depth, and from the greedy method in [13], [14]. It can be observed that our proposed method finds solutions that are equal or at least very close to the optimal bit depth allocation. The small deviations are due to (a) the discretization of the solution of (25), and (b) some possible mismatch with the quantization model used in (17). Our method performs better than [13], [14] in some regions, while performing at least as good in the rest. Furthermore, also the computation time is much lower (on 100 Monte Carlo runs with $K=32$ sensors, the ratio between the computing times $t_{\text {greedy }} / t_{\text {proposed }}$ is 64.23 on average, with a standard deviation of 63.69).

We are now interested in the energy savings with respect to homogeneous allocation, given by

$$
\xi=100 \cdot\left(1-E_{\mathrm{opt}} / E_{\mathrm{homog}}\right),
$$

where $E_{\mathrm{opt}}$ and $E_{\mathrm{homog}}$ are the total communication energy across all sensors corresponding respectively to the solution of (25) and to homogeneous allocation. Figure 2 shows the energy savings $\xi$ from 100 scenarios with different numbers of sensors and three noise sources where, in each scenario, (25) is solved with the same MMSE threshold $\eta$ as the corresponding homogeneous allocation, from 12 to 2 bits. It can be readily observed that the energy savings are significant in most scenarios.

\section{CONCLUSIONS}

We have studied a bit depth allocation problem for multichannel signal estimation in a WSN, which aims at minimizing the energy consumption while satisfying an MMSE constraint. We have shown how, for uniform quantization, an application of the matrix inversion lemma allows to transform the a priori non-convex MMSE constraint into a convex constraint even in the presence of correlated noise. As long as the energy model is also convex, this guarantees the global optimality of the solution up to discretization. Finally, we have demonstrated through numerical simulations that our method indeed finds the (near-) optimal solution, and that significant energy savings can be achieved with respect to a homogeneous allocation. 


\section{REFERENCES}

[1] G. Anastasi, M. Conti, M. Di Francesco, and A. Passarella, "Energy conservation in wireless sensor networks: A survey," Ad Hoc Networks, vol. 7 , no. 3, pp. 537 - 568, 2009.

[2] S. Doclo, A. Spriet, J. Wouters, and M. Moonen, "Frequency-domain criterion for the speech distortion weighted multichannel Wiener filter for robust noise reduction," Speech Communication, vol. 49, no. 7-8, pp. $636-656,2007$, Speech Enhancement.

[3] B. Cornelis, M. Moonen, and J. Wouters, "Performance analysis of multichannel Wiener filter-based noise reduction in hearing aids under second order statistics estimation errors," IEEE Transactions on Audio, Speech, and Language Processing, vol. 19, no. 5, pp. 1368-1381, July 2011.

[4] S. Markovich-Golan, S. Gannot, and I. Cohen, "Distributed multiple constraints generalized sidelobe canceler for fully connected wireless acoustic sensor networks," IEEE Transactions on Audio, Speech, and Language Processing, vol. 21, no. 2, pp. 343-356, Feb 2013.

[5] A. Hassani, A. Bertrand, and M. Moonen, "Cooperative integrated noise reduction and node-specific direction-of-arrival estimation in a fully connected wireless acoustic sensor network," Signal Processing, vol. 107 , pp. $68-81,2015$.

[6] A. Bertrand, "Distributed signal processing for wireless EEG sensor networks," IEEE Transactions on Neural Systems and Rehabilitation Engineering, vol. 23, no. 6, pp. 923-935, 2015.

[7] J.-J. Xiao, S. Cui, Z.-Q. Luo, and A. J. Goldsmith, "Power scheduling of universal decentralized estimation in sensor networks," IEEE Trans. on Signal Processing, vol. 54, no. 2, pp. 413-422, Feb 2006.

8] A. Sani and A. Vosoughi, "On distributed linear estimation with observation model uncertainties," IEEE Transactions on Signal Processing, vol. 66 , no. 12 , pp. 3212-3227, June 2018 .

[9] A. Krasnopeev, Jin-Jun Xiao, and Zhi-Quan Luo, "Minimum energy decentralized estimation in sensor network with correlated sensor noise," in Proceedings. (ICASSP '05). IEEE International Conference on Acoustics, Speech, and Signal Processing, 2005., vol. 3, March 2005, pp. iii/673-iii/676 Vol. 3.

[10] M. H. Chaudhary and L. Vandendorpe, "Power constrained linear estimation in wireless sensor networks with correlated data and digital modulation," IEEE Transactions on Signal Processing, vol. 60, no. 2 pp. 570-584, Feb 2012.

[11] Y. Huang and Y. Hua, "Energy planning for progressive estimation in multihop sensor networks," IEEE Transactions on Signal Processing, vol. 57, no. 10, pp. 4052-4065, Oct 2009.

[12] Y. Huang and Y. Hua, "On energy for progressive and consensus estimation in multihop sensor networks," IEEE Transactions on Signal Processing, vol. 59, no. 8, pp. 3863-3875, Aug 2011.

[13] F. de la Hucha Arce, F. Rosas, M. Moonen, M. Verhelst, and A. Bertrand, "Generalized signal utility for LMMSE signal estimation with application to greedy quantization in wireless sensor networks," IEEE Signal Processing Letters, vol. 23, no. 9, pp. 1202-1206, Sept 2016.

[14] F. de la Hucha Arce, M. Moonen, M. Verhelst, and A. Bertrand, "Adaptive quantization for multichannel Wiener filter-based speech enhancement in wireless acoustic sensor networks," Wireless Communications and Mobile Computing (Special Issue in Wireless Acoustic Sensor Networks and Applications), vol. 2017, no. Article ID 3173196 , 15 pages, 2017.

[15] J. Zhang, R. Heusdens, and R. C. Hendriks, "Rate-distributed spatial filtering based noise reduction in wireless acoustic sensor networks," IEEE/ACM Transactions on Audio, Speech, and Language Processing, vol. 26, no. 11, pp. 2015-2026, Nov 2018.

[16] R. Serizel, M. Moonen, B. V. Dijk, and J. Wouters, "Low-rank approximation based multichannel Wiener filter algorithms for noise reduction with application in cochlear implants," IEEE/ACM Transactions on Audio, Speech, and Language Processing, vol. 22, no. 4, pp. 785-799, April 2014.

[17] A. Hassani, A. Bertrand, and M. Moonen, "GEVD-based low-rank approximation for distributed adaptive node-specific signal estimation in wireless sensor networks," IEEE Transactions on Signal Processing, vol. 64, no. 10, pp. 2557-2572, May 2016

[18] A. Sripad and D. Snyder, "A necessary and sufficient condition for quantization errors to be uniform and white," IEEE Transactions on Acoustics, Speech, and Signal Processing, vol. 25, no. 5, pp. 442-448, Oct 1977.

[19] R. M. Gray, "Quantization noise spectra," IEEE Transactions on Information Theory, vol. 36, no. 6, pp. 1220-1244, Nov 1990.

[20] R. M. Gray and D. L. Neuhof, "Quantization," IEEE Transactions on Information Theory, vol. 44, no. 6, pp. 2325-2383, Oct 1998.
[21] S. Shah and B. Beferull-Lozano, "Adaptive quantization for multihop progressive estimation in wireless sensor networks," in 21st European Signal Processing Conference (EUSIPCO 2013), Sep. 2013, pp. 1-5.

[22] R. A. Horn and C. R. Johnson, Matrix Analysis, 2nd ed. Cambridge University Press, 2013

[23] S. Boyd and L. Vandenberghe, Convex Optimization. Cambridge University Press, 2004

[24] M. Grant and S. Boyd, "CVX: Matlab software for disciplined convex programming, version 2.1," http://cvxr.com/cvx, Mar. 2014

[25] _ "Graph implementations for nonsmooth convex programs," in Recent Advances in Learning and Control, ser. Lecture Notes in Control and Information Sciences, V. Blondel, S. Boyd, and H. Kimura, Eds. Springer-Verlag Limited, 2008, pp. 95-110. 


\section{Supplementary text material}

Proof of the condition (26): The cost function of (25) for $E_{k}\left(b_{k}\right)=\rho_{k}\left(2^{\alpha b_{k}}-1\right), \alpha>0$, $q \geq 1, \rho_{k} \geq 0 \forall k$, is given by

$$
E_{\alpha, q}\left(b_{1}, \ldots, b_{K}\right)=\sum_{k=1}^{K}\left(E\left(b_{k}\right)\right)^{q}
$$

We want to find the values of $\alpha$ and $q$ for which $\left(\tilde{E}_{k}\left(u_{k}\right)\right)^{q}=\left(E_{k}\left(2^{-1} \log _{2} u_{k}\right)\right)^{q}$ is convex, which implies the convexity of $\tilde{E}_{\alpha, q}\left(u_{1}, \ldots, u_{K}\right)$ since the latter would be a sum of convex functions. In the following we drop the index $k$ in order to ease the notation. We define

$$
f(u)=\rho^{q}\left(u^{\frac{\alpha}{2}}-1\right)^{q}, \quad \operatorname{Dom} f=\{u \in \mathbb{R}: u \geq 1\},
$$

which is equal to $\left(\tilde{E}_{k}\left(u_{k}\right)\right)^{q}$ for any index $k$. We know that $f(u)$ is convex on Dom $f$ if and only if its second derivative $f^{\prime \prime}(u)$ is non-negative ${ }^{1}$ in $\operatorname{Dom} f$, i.e., $f^{\prime \prime}(u) \geq 0, \forall u \geq 1$. The first derivative $f^{\prime}(u)$ is given by

$$
f^{\prime}(u)=\rho^{q} q \frac{\alpha}{2}\left(u^{\frac{\alpha}{2}}-1\right)^{q-1} u^{\frac{\alpha}{2}-1} .
$$

The second derivative $f^{\prime \prime}(u)$ is then given by

$$
\begin{aligned}
f^{\prime \prime}(u) & =\rho^{q} q \frac{\alpha}{2}\left\{(q-1)\left(u^{\frac{\alpha}{2}}-1\right)^{q-2} \frac{\alpha}{2} u^{\alpha-2}+\left(u^{\frac{\alpha}{2}}-1\right)^{q-1}\left(\frac{\alpha}{2}-1\right) u^{\frac{\alpha}{2}-2}\right\} \\
& =\rho^{q} q \frac{\alpha}{2}\left(u^{\frac{\alpha}{2}}-1\right)^{q-2}\left\{(q-1) \frac{\alpha}{2} u^{\alpha-2}+\left(u^{\frac{\alpha}{2}}-1\right)\left(\frac{\alpha}{2}-1\right) u^{\frac{\alpha}{2}-2}\right\} \\
& =\rho^{q} q \frac{\alpha^{2}}{4}\left(u^{\frac{\alpha}{2}}-1\right)^{q-2}\left\{(q-1) u^{\alpha-2}+\left(1-\frac{2}{\alpha}\right)\left(u^{\alpha-2}-u^{\frac{\alpha}{2}-2}\right)\right\} \\
& =\rho^{q} q \frac{\alpha^{2}}{4}\left(u^{\frac{\alpha}{2}}-1\right)^{q-2} u^{\alpha-2}\left\{(q-1)+\left(1-\frac{2}{\alpha}\right)\left(1-u^{-\frac{\alpha}{2}}\right)\right\} .
\end{aligned}
$$

Note that in (34) all the factors to the left of the curly braces are strictly positive for $u \geq 1$ and any $\alpha>0$ and $q \geq 1$. Then the condition for the convexity of $f(u)$ in (29) can be written as

$$
\forall u \in \operatorname{Dom} f:(q-1)+\left(1-\frac{2}{\alpha}\right)\left(1-u^{-\frac{\alpha}{2}}\right) \geq 0 .
$$

Since $u \geq 1$, it follows that $0 \leq 1-u^{-\frac{\alpha}{2}}<1, \forall \alpha>0$. Therefore, as $\alpha>0$ and $q \geq 1$, condition (35) is equivalent to

$$
(q-1)+\left(1-\frac{2}{\alpha}\right) \geq 0
$$

which is equivalent to

$$
q \geq \frac{2}{\alpha}
$$

which is the condition for the convexity of $\tilde{E}_{\alpha, q}\left(u_{1}, \ldots, u_{K}\right)$, the cost function of (25).

\footnotetext{
${ }^{1}$ The requirement of $\operatorname{Dom} f$ being convex is already satisfied by $u \geq 1$.
} 